\title{
HIGH TEMPERATURE CYCLIC TEST RIG - A NEW RIG FOR HOT GAS PARTS LIFETIME VALIDATION
}

\author{
Wieland Ammer ${ }^{a *}$, Mario Raddatz $^{a}, \mathrm{Uwe} \mathrm{Gampe}^{a}, \mathrm{Julien} \mathrm{Nussbaum}^{b}$, \\ Uwe Ruedel ${ }^{b}$, Rudolf KellereR ${ }^{b}$ \\ ${ }^{a}$ TU Dresden, Institute of Power Engineering, Faculty of Mechanical Engineering, Chair of Thermal Power \\ Machinery and Plants, 01062 Dresden \\ ${ }^{b}$ Ansaldo Energia Switzerland, Roemerstrasse 36, 5401 Baden \\ * corresponding author: wieland.ammer@tu-dresden.de
}

\begin{abstract}
Flexible gas turbine operation requires adapted hot gas parts design and lifetime assessment methods. The High Temperature Cyclic Test Rig (HTCTR) is developed to cyclically test internally cooled components from combustor and turbine sections of gas turbines in close-to-engine conditions for a wide range of operating conditions in order to improve component lifetime management. These advanced tests should serve as basis for new model development and validation for conditions that are more representative of those in an engine than the standard material specimen tests.
\end{abstract}

KEYWORDS: Turbine, cyclic, lifetime.

\section{INTRODUCTION}

The European Commission's broader energy and climate objectives focus on several cost-efficient measures to make the European economy more climate-friendly, more energy efficient and less energy consuming. For the integration of renewable energy sources (RES) into the total energy mix for power generation, the targets foresee a reduction of the greenhouse gas emissions by 2030 to $40 \%$, by 2040 to $60 \%$ and by 2050 to $80 \%$ below 1990 levels, respectively [1]. However, due to the intermittent nature of the RES, and in the absence of large scale energy storage technologies with a sufficiently high technological and economical maturity level, the existing conventional thermal power plants are not capable of offering the required operational flexibility without impeding their efficiency and engine lifetime through increased wear and damage induced by the higher number of (shorter) operating/loading cycles.

For the introduction of new technologies to balance demand peaks with renewable output fluctuations at minimal fuel consumption and emissions without negative effects on cycling operation, the FLEXTURBINE consortium is part of the EU Horizon2020 activities and aims to develop medium-to-long term solutions for the implementation into existing and new power plants. The consortium consists of the main European turbine manufacturers, renowned research institutes and universities.

\section{AIMS}

The present work describes the strategy and the concept for the development of a high temperature cyclic test rig. The purpose of this rig is the validation of hot gas parts at engine relevant conditions to increase the overall confidence level in the lifetime predictions.
The test rig will be used for the cyclic testing of novel cooling designs, thermal barrier coatings (TBC), materials and for the validation of lifetime methods.

New technologies can be tested in the test rig like:

- Optimized geometries allowed by manufacturing techniques like Selective Laser Melting process

- Thermal barrier coatings where both cyclic life and erosion can be tested

- New sealing concepts at relevant temperature and pressure

The target is to apply cyclic thermal loads to test samples that correspond to current and future gas turbine design targets. The temperature difference between cold and hot steady state has to be high enough to achieve the sample failure (or at least crack initiation) within a reasonable number of cycles/testing time. Target value for crack initiation failure is after 500 cycles, corresponding to approximatively 2 weeks. HTCTR will enable the possibility to test components of current and future gas turbines at a TRL level (TRL5) which could not yet be tested.

As in the engine, thermal loading of the samples is generated by a hot gas flow which overflow the sample surface. The temperature of the hot gas side as well as the through-the-wall temperature have therefore to be reproduced as close as possible to the engine conditions. In order to avoid the wrong interpretation of results in case of crack initiation, the transient damaging effects have to be negligible compared to hot to cold steady states damages.

In Figure 1 a qualitative course of a cycle is illustrated by plotting the hot gas temperature $\mathrm{T}_{\mathrm{HG}}$ and the heat transfer coefficient $\mathrm{HTC}_{\mathrm{HG}}$ on the hot gas surface. At the beginning of each cycle both variables increase for around three minutes until a maximum 


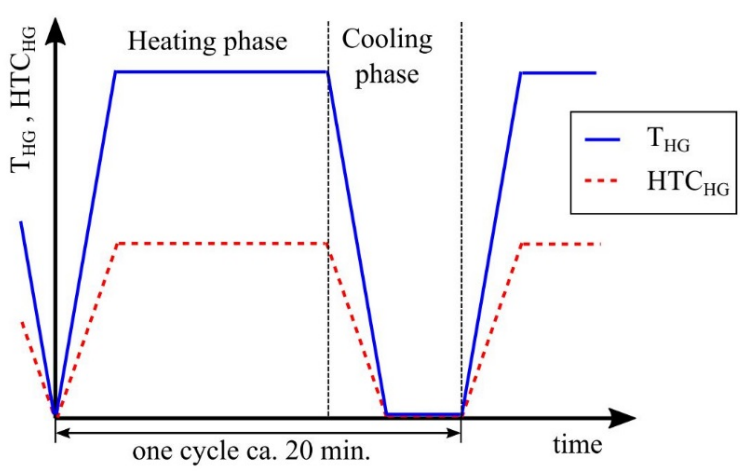

Figure 1. Qualitative sketch of hot gas temperature and heat transfer coefficient on hot gas side.

value is achieved. This state is maintained several minutes to realize sufficient steady state conditions in the test sample. Following this, hot gas temperature and heat transfer coefficient are decreased to a minimum level with a subsequent second cold steady state.

The measurements of boundary conditions via the proper instrumentation will be used to calibrate the Finite-Element calculations. In a second stage, a low cycle lifetime assessment will be performed to estimate the number of cycles until crack initiation based on current lifing models. The cyclic test will be performed with regular borescope inspection to monitor expected life limiting location and detect cracks. The end of the test is defined as the failure of the test sample, which is not possible in a real turbine. The observed number of cycles to crack initiation and sample failure will then be compared to numerical results and lifing models will be adapted accordingly.

\section{Concept And VAlidation}

\subsection{CONCEPT}

In order to fulfil the hot gas generation requirements, an enhanced FLOX burner [2] is employed adapted and provided by the Institute of Combustion Technology of DLR Stuttgart. The target is to avoid the cyclic operation of the burner since the burner is expected to survive several test campaigns. Therefore the concept of the test rig HTCTR architecture has been developed in the form to enable the continuous operation of the burner.

Downstream of the air-cooled combustion chamber, the hot gas path is split into two channels containing one test sample each, meaning that two samples per campaign can be tested in parallel and leading to a significant costs decrease (the test samples can be different). Downstream to the test section, a rotating valve will be used to generate the cycles: during the heating phase, the valve is kept open whereas during the cooling phase the valve is almost closed and purge air is injected upstream to the specimen. While one sample is heated, the other one is cooled down. During the course of the test period both hot gas paths are alternately opened and closed to realize thermal cycles. The angle of the control valve and their rotating velocity are selected in the form to prevent negative impacts on the FLOX burner stability.

All described components belonging to the inner housing are thermal highly loaded by the internal hot gas flow. To prevent an additional loading caused potential by the pressure requirements the inner housing is surrounded by a pressure vessel.

The selected concept is presented in Figure 2.

\subsection{Detailed Design}

The detailed design of the test rig components has almost been completed. As a first step, several 1D thermodynamic simulations have been performed in order to estimate the flame temperature required to achieve the relevant thermal boundary conditions at test section and the different heat loss to the components along the hot gas path.

The burner used in the test rig is a modified version of the FLOX burner from DLR Stuttgart. The design has been slightly modified to achieve the operating point required to generate the cycles. The validation of the new design has been supported by experiments in the HBK-S test facility from DLR Stuttgart. Since the pressure in the HBK-S test cell is limited to a lower value than the one required in the HTCTR, CFD calculations were used to 1 . calibrate the model at low pressure based on experimental results 2 . extrapolate the operation of the burner to higher temperatures. Different configurations have been tested and/or simulated (flame temperature, air/fuel ratio, combustion chamber length, hot gas path geometry) in order to better characterize the behavior of the burner. The main uncertainty remains in the stability of the burner which is difficult to capture with CFD calculations. The CFD results have been then used as boundary conditions for the design of all other hot gas path components.

The combustion chamber consists of an inner liner made of Hastelloy-X material and an outer liner made of STS18/8 material. The chamber is air-cooled and specific focus on cooling features like cooling ribs have been made. Due to the high heat inputs expected from the combustion, the inner liner will be TBC coated.

All other hot gas path components are made of $\mathrm{CuCr} 1 \mathrm{Zr}$ alloy and are intended to be intensively water-cooled. For each component, hot gas side boundary conditions have been extracted from the main CFD calculations of the hot gas path. Then an iterative process has been used to optimize the cooling structure of the components, mainly by implementing cooling holes or ribs in the components, with the support of CFD calculations. In order to validate the design of the components, a FE calculation has been 


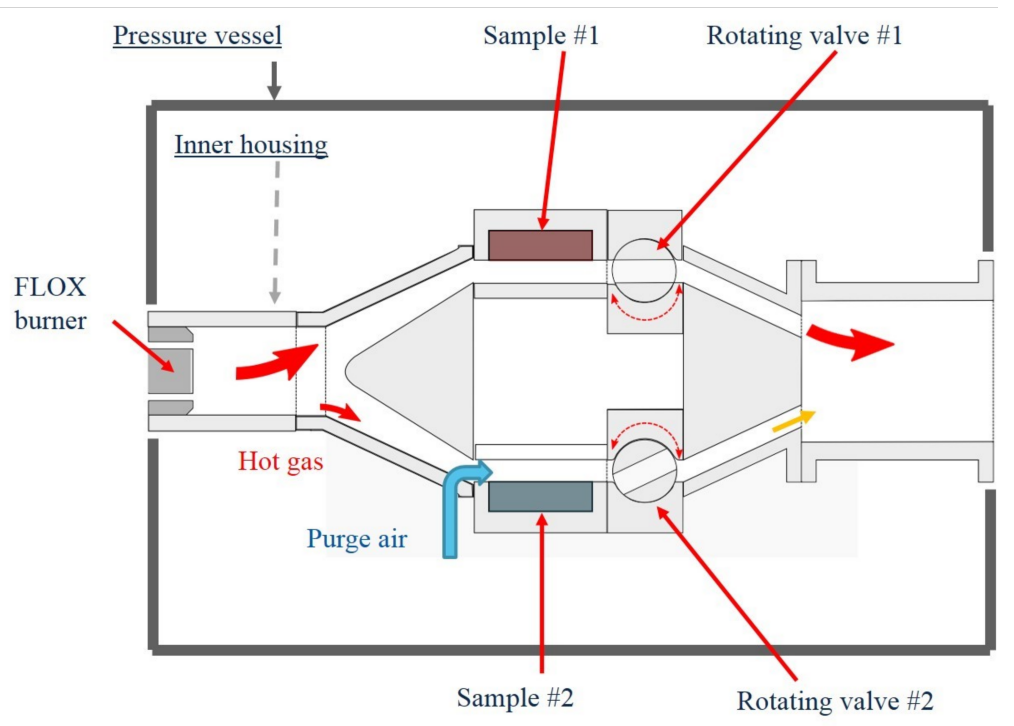

Figure 2. Test section concept.

performed to get the temperature and stress distributions in the components as well as at the interfaces. A lifetime assessment has been then performed to insure the integrity of the components for at least five test campaigns.

The pressure vessel is also designed based on CFD and FE calculations. During test operation a slightly higher pressure will be maintained in the outer housing than in the hot gas path in order to avoid hot gas leakage. Since this component will be under pressure during operation and in direct contact with the ambient atmosphere, the design will be cross-checked by a technical supervisor.

In order to guarantee the proper operation of the test rig, each components have been designed using conservative assumptions on boundary conditions and material properties.

Figure 3 illustrates the structure of the test rig in form of an axial cut.

\subsection{INSTRUMENTATION}

Intensive instrumentation is planned in the test rig

(1.) to get relevant scientific information of the temperature distribution and information about the boundary conditions of the test samples

(2.) to guarantee the safety of the test rig in case of unexpected events like seal failure

(3.) to operate the test rig according to the operating concept and cyclic loading

In total, approximately hundred measurement points are planned in the whole test rig. 40 of them are dedicated to the test samples and their boundary conditions. The main difficulty is to measure the hot gas temperature at test section, where the conditions (temperature and mass flow) are extreme. A concept with correlated measurements from thermocouples and pyrometer is under development to get such information, at least during the hot commissioning. For the first validation test, only metal temperature on both hot gas and cooled sample sides will be measured. No stress or strain measurements are planned, but thermal strains will be estimated based on calibrated thermal field.

In the combustion chamber, a dynamic measurement sensor is required to monitor the pulsation of the burner, since the stability of the burner has been identified as high risk. During hot commissioning, it is planned to test different operating points in order to determine the best set of parameters.

The other measurement points are mainly metal temperature for protection system, as well as inlet/outlet temperature, pressure and/or mass flow for each medium supply line.

\subsection{Adaptation to HBK-3 test Cell}

The target is to ship and assemble the test rig in the HBK-3 test cell at the Institute of Propulsion Technology of DLR Cologne. HBK-3 provides the sufficient infrastructure to operate the test rig: (pre-heated or not) air and water, fuel, and data acquisition system. Adaptation of the HBK-3 facility to the test rig is ongoing in cooperation with DLR Cologne. It has been scheduled that test infrastructure design is completed in October 2018 and a draft data acquisition system with few measurements points from HBK-3 ready.

\subsection{VALIDATION STRATEGY}

The first milestones are the cold assembly and leakage tests, currently planned for autumn 2018. All the components will be shipped to DLR Cologne (Germany) where the test rig will be assembled in the HBK-3 facility. For this first step, the TBC and the instrumentation won't be applied to the parts. Cold leakage tests at relevant pressure levels will be performed and the rig component design will be adjusted if required. 


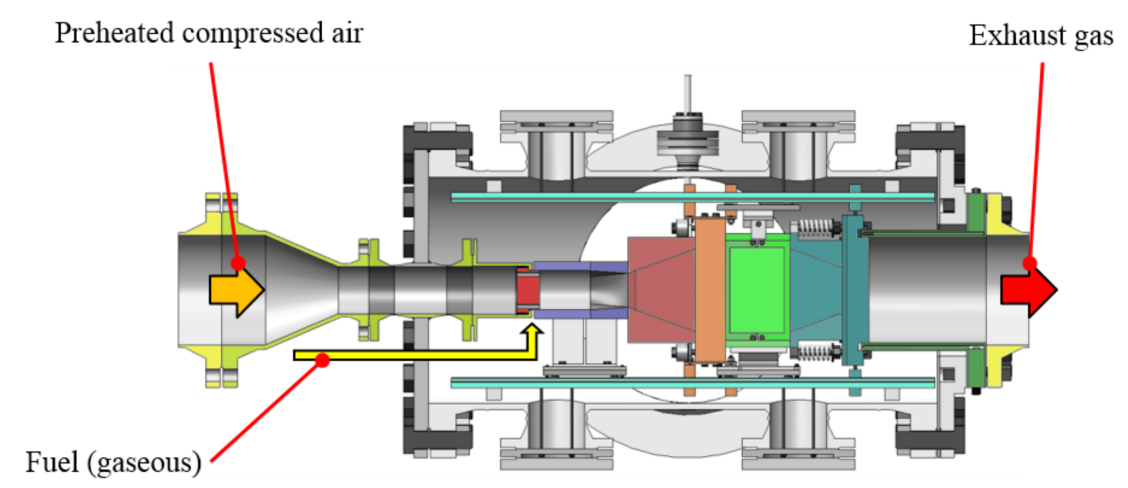

Figure 3. Axial cut of HTCTR design.

After the disassembly of the test rig, the coating will be applied on the hot gas components and the instrumentation will be implemented.

The second milestone is the final validation campaign in May 2019 where the hot commissioning with the entire instrumentation and the test campaign on a stator heat shield will be performed. Post-processing will follow and results become available before the end of the project in June 2019.

\section{Discussion}

The high test rig parameters for engine close conditions are representing a real challenge for rig design and operation.

The difference between hot and cold steady states has to be high enough in order to achieve crack initiation at the test sample within a short number of cycles. CFD and FE calculations are used to evaluate the two steady states and the associated expected lifetime. In case the measurements done during the hot commissioning don't show sufficiently high temperature differences, the cooling medium supply parameters will be adapted by decreasing cooling in some components and increasing cooling at test sections.

Due to thermal expansion, sealing of two neighboring components is challenging. Currently, different sealing concepts are under investigation depending on the interface:

- metal seals like E-seals or strip seals

- polymer seals with higher ductility

Cold leakage test is planned to validate the concepts in cold state. In case some seals fail, the design will be improved before the final validation test campaign in May 2019. Of course the hot operation will lead to larger deformations so that remaining uncertainties will be clarified with the first test campaign.

Finally, the control valve is a critical component. It is supposed to generate the cycles by opening and closing the hot gas path and its failure (e.g. due to sticking) will lead to the end of test campaign and the replacement of the control valve housing and/or rotating cylinder. The design of the control valve is still under progress and two options have been selected, either with an intensively cooled design based on copper alloy or with an uncooled design based on high refractory ceramic material.

\section{Conclusions}

The HTCTR is aimed to support the turbine's hot gas parts design with novel, easier and cheaper testing capabilities on component level for a wide range of current and future close-to-engine conditions. Design solutions of the rig components based on extensive detailed heat balance calculations and fluid-structure interaction finite element analyses. Particular challenges for all partners were ensuring compliance with infrastructural limits regarding cooling water and compressed air. HTCTR developement is still ongoing and the first cold assembly is planned before the end of the year. The first test campaign is expected during spring next year and results will be available for the middle of 2019 .

\section{ACKNowledGements}

This project has received funding from the European Unions Horizon 2020 research and innovation programme under grant agreement No. 653941.

This work was supported by the Swiss State Secretary for Education, Research and Innovation (SERI) under contract number 18.00008 .

The opinions expressed and arguments employed herein do not necessarily reflect the official views of the Swiss Government.

\section{REFERENCES}

[1] The European Commission. The European Commission's Priorities, Energy union and climate, 2050 low-carbon economy, 2018. https://ec.europa. eu/clima/policies/strategies/2050_en

[2] O. Lammel, H. Schütz, G. Schmitz, et al. FLOX ${ }^{\circledR}$ Combustion at High Power Density and High Flame Temperatures. Journal of Engineering for Gas Turbines and Power 132(12), 2010. DOI:10.1115/1.4001825. 